\title{
HUMAN-ROBOT COLLABORATION: IDENTIFYING THE CHALLENGES OF MANUFACTURING SYSTEM INTEGRATION
}

\author{
MICHELLE KELLY HECKLES and SARAH R FLETCHER \\ School of Aerospace, Transport and Manufacturing (SATM), Cranfield University, \\ Cranfield, MK43 OAL, United Kingdom \\ E-mail: michelle.k.heckles@cranfield.ac.uk, s.fletcher@cranfield.ac.uk \\ www.cranfield.ac.uk
}

Due to incredible advancements in the processes of manufacturing, the world is facing a fourth industrial revolution focusing on the development of cyber-physical systems. Human-robot collaboration will be an integral part of these new systems. In the conventional manufacturing process humans and robots have been kept separate from each other to prevent serious harm coming to the human operators. However, considerable advancements in technology have introduced large-scale robotics that are able to successfully and safely interact with human workers, which we will soon see being deployed across the manufacturing industry, to work alongside teams of human operators to improve many aspects of the production process such as speed and uniformity. These developments will not only revolutionise the processes of manufacturing, but will also have a considerable impact on the humans that will be working alongside them. These changes could have physical, mental and emotional impacts on human operators who will likely experience considerable changes to the way they work. In this paper we will discuss the current challenges facing human-robot collaboration integration, worker needs, acceptance, trust, and other ethical issues concerning what changes may need to be made for the successful integration of collaborative robotics, and how this research will identify the human factors that need to be incorporated within future risk assessments/systems integration.

\section{Introduction}

Over the course of the past 300 years we have experienced many industrial and technological revolutions that have completely changed the processes of manufacturing, from being a fully manual industry relying heavily on physical labour during the first industrial revolution, to the most recent industrial revolution and the rise of the 'digital age' which has brought massive changes to 
the way we manufacture, design and use consumer products. Nonetheless, despite the ongoing additions and improvements that digitalisation continues to make, we are now moving towards a new industrial revolution, which at its heart is an amalgamation of all the technologies that have come before being used in new and novel ways, to make improvements to production costs, capabilities and working environments (1). This new industrial revolution has been labelled as 'Industry 4.0' (2), which will see the manufacturing environments improved by not only digital technologies, but also by the development of collaborative robotics (3).

Traditional manufacturing processes will be disrupted with the advent of new intelligent systems, and increasingly fluid and dynamic cyber-physical systems will begin to be used across the manufacturing industry (4). With these changes will come optimised, data-driven production allowing for intelligent interactions between humans and collaborative robots, which will be able to be developed for multiple purposes on factory floors (5), therefore allowing for a more significant level of integration between human workers and their autonomous counterparts. However, this could have a serious impact on those human workers.

Technological advancements are impacting almost all areas of human life, including the workplace. Human workers will need to be able to cope with these changes, and adapt as quickly as possible to the increasing levels of intelligent automation that will become standard across areas of the manufacturing industry. To allow a safe and easy transition into this new way of working, we need to consider how human-robot collaboration will affect workers both physically and mentally. Developing systems to successfully integrate collaborative robots into teams of human workers in a way that they can accept and utilise these new tools. With the increased global usage of robotics in the manufacturing industry, it is more important than ever to understand what changes will be affecting our future workplaces.

This paper will discuss the research that needs to be performed to discover the human factors that should be incorporated into future systems integration and risk assessments for Human-robot collaboration, particularly in regards to the present state of industrial level human-robot collaboration. These studies will allow us to further understand the factors that most affect the human workers acceptance of collaborative robots. To improve worker acceptance of robotics, we need to determine how we can train and empower human operators to confidently interact with these new machines. Therefore, we need to develop an understanding of what the workers needs are when it comes to the integration of collaborative robotics, which will allow a greater level of communication within organisations, and help to increase rates of worker acceptance. 


\subsection{Background of Human-Robot Collaboration}

Despite continued introduction of automation to the manufacturing industry, it is understood that the biggest advantage of collaborative robots is in the ability to incorporate both the benefits of automation with the flexibility, soft skills and cognitive capabilities of human workers (6). Human-robot collaboration (HRC), is defined as when humans and robots come into direct contact with one another and form a close physical relationship in order to successfully complete a task (7). Within this dynamic system each component should be capable of observing and estimating the others reactions by processing sensory information (8), allowing for harmony between the human and robot workers. Furthermore, with the ongoing interest the manufacturing industry has shown in the possibility of introducing industrial robots into existing teams of human operators, to improve current performance levels $(9,10)$, we need to understand what steps must be taken for successful implementation.

Industrial collaborative robots have the ability to enhance the efficiency and productivity of the manufacturing process, as the weakness of one team member can be complemented by the capabilities and strengths present in the other (11). Consequentially, when human teammates collaborate on tasks they can achieve a level of coordination that exceeds a single worker. $(12,13)$. Within this research we are interested in how robots could similarly perform more fluently with their human counterparts.

Robots that are commonly used today have generally been separated from the human workforce due to potentially physical dangers associated with the strength and movement speeds presented by large scale manufacturing robotics (14). This has meant that within the factory layout, robotics have been placed in positions that make use of their capabilities, such as for the unskilled, repetitive tasks that require no level of mental flexibility or intelligence (15), which leaves more complicated tasks to the human workers. In conjunction to this, traditional robotic installations have some weaknesses when compared to manual operators, such as a lack of critical thinking and problem solving skills. These issues would often lower efficiency and usability, to run at optimal levels. An example of this is the often costly safety equipment required to avoid injury to humans on the factory floor. This is especially true for the more powerful large scale robots, which required safety barriers, fencing or sensor-based light curtains to be placed around them to prevent any injury to human workers (16), but this can often cause costly disruptions and decrease work efficiency. However, collaborative robotics require much less safety equipment, which will allow the robots to work in a more freed and open style where they can share their workspace with a human, or team of humans (17). As both humans and robots offer different strengths and skills, combined utilization of the two could allow robot installation to become truly competitive, particularly if the more difficult robot operations are able to be 
managed in a more cost effective nature. Additionally, collaborative robots can improve working conditions by performing the dangerous, repetitive and heavy tasks that can cause injury and health problems to human workers. For example, musculoskeletal disorders are a serious health concern for workers in developed countries, with around half the workforce affected (18). Introducing collaborative robots into the workforce could considerably improve the physical health of factory workers. Furthermore, the use of collaborative robots could prove to be beneficial as they can be managed and taught through intuitive systems such as, walk-through programming or augmented reality $(19,20)$, with greater ease. In comparison, traditional robots often require expert engineers to reprogram them when tasks change or develop.

The idea of coupling human workers with robots to benefit from the strengths of both is not a new concept. With the continued advancements in the way we monitor the safety and efficiency of workspaces, collaborative robotics could be introduced as a key component with much greater ease (21). Theoretically, these new collaborative systems should be much safer than current safety guarding setups and will enable workers to work more directly with future manufacturing robotics.

\section{Current Challenges Facing Human-Robot Collaboration}

Although we have seen a rapid growth in the use of automated systems in the manufacturing industry, the significant role that humans play in the production chain cannot be ignored. Many tasks and processes within manufacturing still require the adaptability and ingenuity of human operators who can react according to their environment far more efficiently than currently shown by robots. Frequent product changeovers, and highly specialised tasks are two examples of areas that are currently seen as unviable for automation as they require dexterity and flexibility that is currently only possible when using human operators (22).

Ordinarily, within the manufacturing industry there has been a prioritisation of technological advancement over the need to understand how an evolving workplace is affecting human workers, especially in regards to their mental and emotional wellbeing. This is especially the case when looking at human-robot collaboration, where evidence suggests that there has been a considerable lack of scrutiny paid towards user issues, which are often the source of failure in collaborative robotics implementation (23). These issues have spurred an interest in understanding the human factors that can contribute to a more effective system (24). In general, current systems are still broadly designed without any true attention given to developing a balanced approach to human users, due to the fact that there is a fundamental lack of understanding of the psychological principles that govern the application of human factors processes (25), this is especially true of Systems integrators who are usually responsible for implementing new 
technologies into the manufacturing workspace. However, currently there is a notable absence of tools or frameworks for systems integrators to use in the implementation of collaborative robotics, primarily due to limited research in the area of worker acceptance and trust.

Inevitably, there will be a need to consider the implications of introducing robots into the workforce, as it has been shown that advanced automation does not completely replace the need for the human operator (26). Although recent research has begun to establish the primary factors that are likely to have an influence on the implementation of collaborative robotics at the organisational and individual level (27), this research has limits to its use in an applied context. Consequently, research into the issues affecting human workers will continue to fall behind in comparison to the speed at which technological advancements are implemented, this is usually the result of overlooking human issues (28). Therefore, if we are to improve manufacturing processes there needs to be deeper considerations given to the design of robotics integration systems.

\subsection{The Human Worker}

Inattention to designing for the human element can be detrimental to the whole manufacturing process as well as the human worker. However, many organisations have not been able to grasp the full potential of these practices. Some reports even suggest that an upper value of $75 \%$ of all implementations have failed in terms of quality, flexibility, and reliability (29). Ironically, the problem does not appear to lie with the machine or the technology itself. Numerous studies have suggested that these practices impose significant organisational challenges and require a fundamental transition in the way business is conducted which in turn is affecting the human element (30). An empirical survey of 759 senior executives of manufacturing organisations (31) concluded that the major barrier towards successful introduction of flexible automated systems is the inattention to human issues.

The extent to which human workers are able to adapt, psychologically and physically, to the continued developments within the workplace, will be one of the most important concerns of the new fourth industrial revolution. It is unavoidable that there will be a sustained growth in the number of robots deployed within the manufacturing industry, however, to prevent the problems that have occurred in many previous technology adoptions (32) from happening again, consideration of the design and usability of collaborative robotics need to be planned for in advance of integration. Moreover, it is likely that the requirements of human 
operators working with collaborative robotics will change dramatically in the future. Although many potential issues may arise from the changes that will occur in the workplace, this research aims to discover and understand the key human factors that may affect the adoption of collaborative robotics.

\subsection{Trust and Acceptance}

A crucial barrier to overcome in the implementation of collaborative robotics is the current lack of trust and acceptance that most factory workers have towards these new machines (33), as they are used to viewing manufacturing robots as being large and dangerous. This may cause workers difficulties when eventually having to interact with the robots, and cause them to actively resist the deployment of the robots onto the shop floor. The key to preventing these issues will be how well workers are able to familiarise themselves with these new machines, and accept them, to some extent, as their co-workers. Consequently, research is being performed concerning issues of acceptance and trust between humans and collaborative robots (34), however, past research has yet to be applied in a work setting. Furthermore, there is still much to understand as such concepts should be carefully introduced, because of the impact they may have to human workers.

Previous research has often focused on the more physical aspects of safety concerns, and ignored the need for research into the needs of workers who may spend long periods of time interacting with these robots $(35,36)$. Additionally, there has also been a lack of consideration for how workers feel about working alongside such machines, especially within the context of psychological language and analysis. Interestingly, we may even have to consider how human operators will change their own behaviours as ever more intelligent and autonomous systems are developed (37). Currently, the focus has been on protecting human workers from physical injury, but we now need to take more considerations on how we can make the transition to a more collaborative workplace as easy as fluid as possible. While current standards have taken aspects of design and social ethics into consideration, they have not always covered the industrial context to which they are most likely to be applied, and therefore have some limitations. Nevertheless, it is clear that organisations need to consider including psychological safeguards at all levels, from initial training to continued workplace support.

To encourage feelings of trust between operators and collaborative robots, could providing in-depth training about the reliability and performance of collaborative systems, allow workers to feel more 
settled? It would be preferable if all operators where made aware of every technical aspect of the system, without anything being held back, which would be a much more ethical approach to take, but is this approach feasible? As of yet there has been no significant research on the level to which workers should be made aware of the true risks of interacting with collaborative robotics. Consequently, failure to attend to the human factors could prove to be detrimental to robotics adoption, and with the concept of industrial HRC still at its infancy, it is crucial to discover and understand the key human factors that need to be considered for the successful implementation of industrial HRC.

\section{Conclusions and Recommendations for the Future}

During this paper we have outlined the current issues facing implementation of collaborative robotics into the manufacturing workspace, and how we can move towards tackling these issues. Despite this, human workers will need to adapt to the evolving workplace, and demonstrate that they are able to adapt to new styles of working, and the likelihood that there current roles within an organisation will change.

Therefore, we must provide a clear system to deliver the information resulting from these future studies. If we are to make an impact in the way systems integrators make decisions, then we must make sure that the research prevents confusion and increases the chances of successful deployment. Additionally, this step will allow organisations to manage the large internal changes that will take place, and allow workers to adapt to new positions naturally. This is arguably the most important future action to be taken, as if the results of the studies are implemented incorrectly or not at all, because of a lack of understanding on the system integrators part, in regards to the language or format that the research is presented in, it could have a significant impact on the success of collaborative robotics integration and therefore, negatively impact an organisation's business. However, we must be aware of the potential limitations that such research can have, as context and environment will play a vital role as to whether it will be successfully applied in a manufacturing setting. Limitations such as; cost efficiency, ethics and reliability could all impact the likelihood of the research being accepted and utilised by the industry.

Overall, prior research has begun to inform us of the psychological impacts of industrial based HRC (25), however there are still many issues to understand. Ideally more time and investment is needed before we can present any definitive system for successful integration. To prevent a continuation of the ignorance surrounding the necessary application of human factors within the HRC 
environment, we must look towards developing a standard system of deployment and integration which can successfully be used by systems integrators, to reach organisational goals and targets in a realistic and sustainable way.

\section{References}

1. Marsh, P. The new industrial revolution: consumers, globalization and the end of mass production. Yale University Press (2012).

2. Hermann, M., Pentek, T., \& Otto, B. Design principles for Industrie 4.0 scenarios: a literature review. Technische Universität Dortmund, Dortmund (2015).

3. Schwab, K. The fourth industrial revolution. Foreign Affairs, 12. (2015).

4. Kagermann, H., Lukas, W., \& Wahlster, W. Industrie 4.0: Mit dem Internet der Dinge auf dem Weg zur 4. industriellen Revolution. VDI nachrichten, 13, (2011).

5. Wan, J., Cai, H., \& Zhou, K. Industrie 4.0: enabling technologies. In Intelligent Computing and Internet of Things (ICIT), 2014 International Conference on (pp. 135-140). IEEE (2015).

6. Ding, Z., \& Hon, B. Constraints analysis and evaluation of manual assembly. CIRP Annals-Manufacturing Technology, 62(1), 1-4 (2013).

7. Krüger, J., Lien, T. K., \& Verl, A. (2009). Cooperation of human and machines in assembly lines. CIRP Annals-Manufacturing Technology, 58(2), 628-646.

8. Lallée,S.,Pattacini,U.,Lemaignan,S.,Lenz,A.,Melhuish,C.,Natale, L., et al. (2012). Towards a platform-independent cooperative human robot interaction system: III an architecture for learning and executing actions and shared plans. IEEE Transactions on Autonomous Mental Development, 4(3), 239-253.

9. Unhelkar, V. V., Siu, H. C., \& Shah, J. A. Comparative performance of human and mobile robotic assistants in collaborative fetch-and-deliver tasks. In Proceedings of the 2014 ACM/IEEE international conference on Human-robot interaction (pp. 82-89). ACM (2014).

10. Schraft, R. D., Meyer, C., Parlitz, C., \& Helms, E. PowerMate-A safe and intuitive robot assistant for handling and assembly tasks. In Robotics and Automation, 2005. ICRA 2005. Proceedings of the 2005 IEEE International Conference on (pp. 4074-4079). IEEE (2005).

11. Bortot D, Born M, Bengler K. Directly or detours? How should industrial robots approach humans?. ACM/IEEE international conference on human-robot interaction (HRI 2013), IEEE, Tokyo (2013).

12. Hoffman, G., \& Breazeal, C. Collaboration in human-robot teams. In AIAA 1st Intelligent Systems Technical Conference (p. 6434) (2004). 
13. You, S., \& Robert, L. Trusting Robots in Teams: Examining the Impacts of Trusting Robots on Team Performance and Satisfaction. In You, S. and Robert, LP (2019). Trusting Robots in Teams: Examining the Impacts of Trusting Robots on Team Performance and Satisfaction, Proceedings of the 52th Hawaii International Conference on System Sciences, Jan (pp. 8-11) (2018).

14. Vasic, M., \& Billard, A. Safety issues in human-robot interactions. In Robotics and Automation (ICRA), 2013 IEEE International Conference (pp. 197-204). IEEE (2013).

15. Hedelind, M. \& Kock, S. Requirements on flexible robot systems for small parts assembly, a case study. Proceedings of the International Symposium on Assembly and Manufacturing, 25-27 May, Tampere, Finland (2011).

16. Kulić, D. \& Croft, E.A. Real-time safety for human-robot interaction. Robotics and Autonomous Systems, 54, 1-12 (2006).

17. Grahn, S., Langbeck, B., Johansen, K., \& Backman, B. Potential advantages using large anthropomorphic robots in human-robot collaborative, hand guided assembly. Procedia CIRP, 44, 281-286 (2016).

18. Schneider, E., \& Irastorza, X. Work-related musculoskeletal disorders in the EU. European Agency for Safety and Health at Work (EU-OSHA) pp, 1184 (2010).

19. Lambrecht, J., \& Krüger, J. Spatial programming for industrial robots based on gestures and augmented reality. In Intelligent Robots and Systems (IROS), 2012 IEEE/RSJ International Conference on (pp. 466472). IEEE 9 (2012)

20. Landi, C. T., Ferraguti, F., Secchi, C., \& Fantuzzi, C. Tool compensation in walk-through programming for admittance-controlled robots. In Industrial Electronics Society, IECON 2016-42nd Annual Conference of the IEEE (pp. 5335-5340). IEEE (2016).

21. Santis, A., \& Siciliano, B. Safety issues for human-robot cooperation in manufacturing systems. Tools and Perspectives in Virtual Manufacturing (2008).

22. Matthias, B., Kock, S., Jerregard, H., Kallman, M., Lundberg, I., \& Mellander, R. Safety of collaborative industrial robots: Certification possibilities for a collaborative assembly robot concept. IEEE International Symposium on Assembly and Manufacturing (ISAM) (pp. 1-6). IEEE (2011).

23. Wang, X. V., Kemény, Z., Váncza, J., \& Wang, L. Human-robot collaborative assembly in cyber-physical production: Classification framework and implementation. CIRP annals, 66(1), 5-8 (2017).

24. Battini, D., Faccio, M., Persona, A., \& Sgarbossa, F. New methodological framework to improve productivity and ergonomics in 
assembly system design. International Journal of Industrial Ergonomics, 41(1), 30-42 (2011).

25. Baxter, G., \& Sommerville, I. Socio-technical systems: From design methods to systems engineering. Interacting with computers, 23(1), 417 (2011).

26. The Economist. Making the future: How robots and people team up to manufacture things in new ways. The Economist, April 21st, 2012. Accessed 30/05/15: http://www.economist.com/node/21552897 (2012).

27. Charalambous, G., Fletcher, S., \& Webb, P. (2015). Identifying the key organisational human factors for introducing human-robot collaboration in industry: an exploratory study. The International Journal of Advanced Manufacturing Technology, 1-13 (2015).

28. King, N., \& Majchrzak, A. Concurrent engineering tools: are the human issues being ignored? IEEE Transactions on engineering management, 43(2), 189-201 (1996).

29. Chung C.A. Human issues influencing the successful implementation of advanced manufacturing technology. Journal of Engineering and Technology Management, 13(3):283-299 (1996).

30. Pun, K. F. Development of an integrated TQM and performance measurement system for self-assessment: a method. Total Quality Management, pp. 13(6), 759-777 (2002).

31. Sheridan, J. H. The new Luddites? Industry week, 239 (no.4), pp. 62-63 (1990).

32. Geels, F. W., \& Smit, W. A. Failed technology futures: pitfalls and lessons from a historical survey. Futures, 32(9-10), 867-885 (2000).

33. Schaefer, K. E., Billings, D. R., Szalma, J. L., Adams, J. K., Sanders, T. L., Chen, J. Y., \& Hancock, P. A. (2014). A meta-analysis of factors influencing the development of trust in automation: Implications for human-robot interaction (No. ARL-TR-6984). ARMY RESEARCH LAB ABERDEEN PROVING GROUND MD HUMAN RESEARCH AND ENGINEERING DIRECTORATE

34. Charalambous, G., Fletcher, S., \& Webb, P. The Development of a Scale to Evaluate Trust in Industrial Human-robot Collaboration. International Journal of Social Robotics, 1-17 (2015).

35. Riek, L. D., Rabinowitch, T. C., Chakrabarti, B., Robinson, P. How anthropomorphism affects empathy toward robots. Proceedings of the 4th ACM/IEEE international conference on Human robot interaction, La Jolla, California, USA, March 09-13, (2009).

36. Wilkinson, C., Bultitude, K., \& Dawson, E. (2011). “Oh yes, robots! People like robots; the robot people should do something": perspectives and prospects in public engagement with robotics. Science Communication, 33(3), 367-397.

37. Chidambaram, V., Chiang, Y. H., \& Mutlu, B. (2012, March). Designing persuasive robots: how robots might persuade people using vocal and 
nonverbal cues. In Proceedings of the seventh annual ACM/IEEE international conference on Human-Robot Interaction (pp. 293-300).

ACM. 